\title{
Cardioprotective effects of tea and its catechins
}

\author{
Nana Li, Yue Zhao, Yuerong Liang* \\ Zhejiang University Tea Research Institute, Hangzhou, China; ${ }^{*}$ Corresponding Author: yrliang@,zju.edu.cn
}

Received 23 February 2013; revised 26 March 2013; accepted 5 April 2013

Copyright (C) 2013 Nana Li et al. This is an open access article distributed under the Creative Commons Attribution License, which permits unrestricted use, distribution, and reproduction in any medium, provided the original work is properly cited.

\begin{abstract}
Tea is rich in polyphenolic catechins which are beneficial to health. There have been evidences suggesting that habitual tea consumption may reduce the risk of cardiovascular disease (CVD). Intake of tea or catechins isolated from tea was shown to inhibit the development of CVD in population studies and in animal models. Many possible pathways and mechanisms were investigated. There have been evidences showing that tea and tea catechins reduced the risk of CVD by enhancing antioxidant activity, attenuating metabolic syndrome, inhibiting angiotensin converting enzyme, improving endothelial dysfunction, preventing cardiac hypertrophy and protecting mitochondria from damage.
\end{abstract}

Keywords: Camellia sinensis; Hypertension; Antioxidant; Metabolic Syndrome; Endothelial Dysfunction; Cardiac Hypertrophy; Mitochondria

\section{INTRODUCTION}

Tea prepared using leaf of Camellia sinensis is one of the most popular beverages worldwide and its habitual consumption has been associated with health benefits. The health beneficial effects of tea are attributed to its polyphenolic catechins [1]. Tea is classified as non-fermented green tea, semi-fermented oolong tea and fullyfermented black tea. Catechins such as (-)-epigallocatechin-3-gallate (EGCG), (-)-epigallocatechin (EGC), (-)epicatechin-3-gallate (ECG) and (-)-epicatechin (EC) are major polyphenols in fresh tea leaf. The tea catechins are oxidized during the fermentation process of tea manufacture, resulting in the formation of orange and red pigments such as theaflavins and thearubigins. Concentration of catechins ranges from $150 \mathrm{mg} / \mathrm{g}$ to $200 \mathrm{mg} / \mathrm{g}$ in green tea, and $40 \mathrm{mg} / \mathrm{g}$ to $60 \mathrm{mg} / \mathrm{g}$ in black tea, with oolong tea in between, owing to their difference in fermentation. The other chemical compositions of tea were also varied among the three kinds of teas (Table 1).

The cardioprotective effects of polyphenols are attributed to antioxidant, anti-inflammatory properties and also improvement of ultra structures of left ventricular mitochondrion and regulation of levels of plasma angiotensins and high density lipoprotein (HDL)-cholesterol. The cardioprotective and antihypertensive effects of tea and its catechins were reviewed and discussed in this paper.

\section{EVIDENCES OF EPIDEMIOLOGICAL AND CASE-CONTROL STUDIES}

An investigation on 1507 subjects (711 men and 796 women) in Tainan City of Taiwan showed that 600 habitual tea drinkers $(39.8 \%$ ) who consumed $120 \mathrm{~mL} / \mathrm{d}$ or more for at least 1 year had significantly lower risk of developing hypertension than nonhabitual tea drinkers. Compared with nonhabitual tea drinkers, the risk of developing hypertension decreased by $46 \%$ for those who drank 120 to $599 \mathrm{~mL} / \mathrm{d}$ and was further reduced by $65 \%$ for those who drank $600 \mathrm{~mL} / \mathrm{d}$ or more after carefully adjusting for age, sex, socioeconomic status, family history of hypertension, body mass index, waist-hip ratio, lifestyle factors, and dietary factors [2]. The Japan Collaborative Cohort Study for Evaluation of Cancer Risk (JACC Study) examined the relationship between the consumption of tea and risk of mortality from cardiovascular disease (CVD), in which 76,979 individuals aged 40 - 79 years free of stroke, coronary heart disease (CHD) and cancer at entry were prospectively followed and the daily consumption of beverages was assessed by questionnaires. It showed that a moderate consumption of

Table 1. Chemical composition of various teas $(\mathrm{mg} / \mathrm{g})$.

\begin{tabular}{cccc}
\hline Component & Green tea & Oolong tea & Black tea \\
\hline Total catechins & $150-200$ & $70-150$ & $40-60$ \\
Caffeine & $20-60$ & $20-50$ & $20-60$ \\
Theanine & $8-20$ & $8-15$ & $5-10$ \\
Theaflavins & - & $3-5$ & $5-20$ \\
Thearubigins & - & $5-20$ & $60-180$ \\
\hline
\end{tabular}


coffee, green tea and oolong tea was associated with a lower risk of mortality from CVD [3].

A study investigating the association between green tea consumption and arteriographically determined coronary atherosclerosis in a Chinese population showed that green tea consumption can protect against the development of artery disease [CAD] in Chinese male patients, with an adjusted odds ratio (OR) of 0.62 (95\% confidence interval, 0.38 - 1.01), compared with those who did not drink green tea. Compared to non-tea drinkers, the ORs were $1.09(0.61-1.96)$ in male patients consuming less than $125 \mathrm{~g}$ of dried green tea leaves per month, $0.36(0.19-0.71)$ for $125-249 \mathrm{~g}$ per month and $0.36(0.17-0.73)$ for $\geq 250 \mathrm{~g}$ per month, with a statistically significant test for trend $(p<0.001)$. Similar doseresponse relationships were also observed for frequency, duration, concentration and starting age of green tea drinking in male patients. However, no inverse association was found between green tea consumption and CAD in female patients [4].

Data from subjects comprised 1827 men and 2918 women aged 20 - 69 years examined by the Korean National Health and Nutrition Examination Survey showed that dietary intake of flavan-3-ols such as tea catechins may have beneficial effects on metabolic syndrome (MetS) risk by reducing the risk of hypertension. In the female subjects, flavan-3-ols intake was inversely associated with the risk of MetS after adjusting for potential confounders, but no significant association between flavan-3-ols intake and risk of MetS was found in the male subjects [5].

\section{ENHANCING ANTIOXIDANT ACTIVITY}

Antioxidant activity of tea depends on tea processing and extraction methods owing to their differences in concentration of antioxidant catechins. Hot water extracts of green tea (catechins concentration $136.40-191.33 \mathrm{mg} / \mathrm{g}$ ) were more effective in antioxidant activity, reducing power and scavenging ability on hydroxyl radicals but less effective in chelating ability on ferrous ions than its cold water extracts (catechins concentration 130.22 $146.28 \mathrm{mg} / \mathrm{g}$ ). The half maximal effective concentration (EC50) values in antioxidant activity and reducing power of green tea extracts were $2.17-2.75 \mathrm{mg} / \mathrm{mL}$ and 0.22 $0.30 \mathrm{mg} / \mathrm{mL}$, respectively, and EC50 values in scavenging ability on hydroxyl radicals and chelating ability on ferrous ions were $3.31-4.54 \mathrm{mg} / \mathrm{mL}$ and $1.63-3.09$ $\mathrm{mg} / \mathrm{mL}[6]$.

Oxidative stress and endothelial dysfunction are closely associated with hypertension and insulin resistance (IR) in metabolic syndrome (MetS). There was study showing that decaffeinated green tea extracts (GTE) reduced the formation of reactive oxygen species (ROS) and nicotinamide adenine dinucleotide phosphate (NADPH) oxi- dase activity, and stimulated phosphorylation of endothelial nitric oxide synthase (eNOS) and Akt in the aorta of a rat model of MetS, which resulted in improved endothelial dysfunction and IR, and eventually lowered blood pressure (BP). When Otsuka Long-Evans Tokushima Fatty (OLETF) rats (13 weeks old, MetS) were supplemented with decaffeinated-GTE $25 \mathrm{mg} / \mathrm{kg}$-day (GTEOLETF), indicators BP $(121 \pm 3 \mathrm{mmHg})$, fasting/postprandial $2 \mathrm{~h}$ glucose $(115 \pm 7 / 132 \pm 16 \mathrm{mg} / \mathrm{dL})$ and insulin level $(2.4 \pm 1.3 \mathrm{ng} / \mathrm{mL})$ of GTE-OLETF were significantly lower than those of OLETF $(\mathrm{BP}=130 \pm 7 \mathrm{mmHg}$, fasting/postprandial $2 \mathrm{~h}$ glucose $=141 \pm 18 / 159 \pm 13$ $\mathrm{mg} / \mathrm{dL}$, insulin $=4.8 \pm 2.3 \mathrm{ng} / \mathrm{mL}$ ). Furthermore, decaffeinated-GTE significantly reduced vascular ROS formation and NADPH oxidase activity, and improved endothelium dependent relaxation in the thoracic aorta of the tested rats. Immunohistochemical staining tests showed that the expression of p47 and p22phox (NADPH oxidase subunits) were suppressed by decaffeinated-GTE, but phosphorylation of eNOS and Akt in the immunoblotting of aortas were stimulated [7].

Though black tea and green tea had different composition of tea polyphenpls, both of them attenuated blood pressure increases through their antioxidant properties in stroke-prone spontaneously hypertensive rats (SHRSP). When SHRSP was fed with black tea polyphenols (BTP, containing $3.5 \mathrm{~g} / \mathrm{L}$ thearubigins, $0.6 \mathrm{~g} / \mathrm{L}$ theaflavins, 0.5 $\mathrm{g} / \mathrm{L}$ flavonols and $0.4 \mathrm{~g} / \mathrm{L}$ catechins) and green tea polyphenols (GTP, containing $3.5 \mathrm{~g} / \mathrm{L}$ catechins, $0.5 \mathrm{~g} / \mathrm{L}$ flavonols and $1 \mathrm{~g} / \mathrm{L}$ polymetric flavonoids) and tap water (control, $30 \mathrm{~mL} / \mathrm{d}$ ), systolic and diastolic BP were significantly lower in the BTP and GTP groups than in the controls during the daytime. The GTP significantly increased catalase expression. The phosphorylated myosin light chain (MLC-p) expression in the aorta was significantly decreased by BTP and GTP [8].

Out-of-control ROS signaling is one of the key events in the pathogenesis of endothelial dysfunction and essential hypertension. Tea polyphenols regulated ROS-related protein expression and might be beneficial in alleviating angiotensin (Ang) II-induced hyperpermeability, preventing endothelial cell dysfunction and development of CVD, including hypertension. Ying et al. found that tea polyphenols decreased the production of ROS via regulation of the protein expression of NADPH oxidase in bovine carotid artery endothelial cells (BCAECs). Both GTP and BTP down-regulated the expression of NADPH oxidase subunits p22phox and p67phox while up-regulating catalase expression $(p<0.05)$. Pre-treatment with GTP or BTP for $24 \mathrm{~h}$ significantly decreased the super-oxide anion level $(\mathrm{p}<0.05)$ and permeable fluorescence intensities in Ang II-stimulated BCAECs [9]. Jówko et al. confirmed that a 4-week supplementation with GTP $(640 \mathrm{mg} / \mathrm{d})$ enhanced the antioxidant de- 
fense system in plasma at rest and, in turn, gave protection against oxidative damage induced by both shortterm muscular endurance test and long-term strength training [10]. Oxidative stress induced by hypertension was reported to cause alterations in neural cytoarchitecture and cognitive dysfunction. Green tea EGCG reduced the progressive increase in blood pressure in spontaneous hypertension rats (SHRs), and prevented most of the increased locomotor activity in addition to improving learning and memory through its powerful antioxidative properties [11].

\section{ATTENUATING METABOLIC SYNDROME}

Metabolic syndrome (MetS) is a group of risk factors that occur together and increase the risk for CVD and diabetes, including insulin resistance (IR), obesity, hypertriglyceridemia, hypercholesterolemia and hypertension.

Hypertension and IR in Sprague-Dawley (SD) rats can be induced by feeding a fructose-rich diet. Wu et al. examined the effects of green tea supplementation $(0.5 \mathrm{~g}$ green tea powder dissolved in $100 \mathrm{~mL}$ of deionized water) on IR and hypertension in the fructose-fed SD rat model. It showed that the fructose group developed fasting hyperglycemia, hyperinsulinemia, and elevated blood pressure. Insulin-stimulated glucose uptake and insulin binding of adipocytes were significantly reduced, and the glucose transporter IV (GLUT IV) content of adipocytes also decreased. All of these metabolic defects, including IR and BP were improved by the green tea supplementation [12]. It is considered that the amelioration of IR by green tea is associated with the increased expression of GLUT IV.

EGCG is considered to augment metabolic and vascular actions of insulin. Like enalapril (an angiotensin converting enzyme inhibitor), acute action of EGCG stimulated the production of nitric oxide from endothelium, resulting in improvement of insulin sensitivity and increase in plasma adiponectin levels in SHR (a model of metabolic syndrome with hypertension, insulin resistance, and overweight) [13].

In a double-blind, placebo-controlled trial, 56 obese, hypertensive subjects were randomized to receive a daily supplement of 1 capsule containing either $379 \mathrm{mg}$ of green tea extract (GTE) or a matching placebo. After 3 months of supplementation, both systolic and diastolic blood pressures had significantly decreased in the GTE group as compared with the placebo group ( $p<0.01)$. Compared with the placebo group, fasting serum glucose, insulin levels, IR, serum tumor necrosis factor and Creactive protein were significantly decreased, whereas total antioxidant status significantly increased in the GTE group ( $<<0.05)$ [14]. IR Improvement by GTE was also observed in SHR test [7].

Hasegawa et al. found that oral administration of powdered green tea $(130 \mathrm{mg} / \mathrm{d})$ to male Zucker rats fed a $50 \%$ sucrose diet containing $15 \%$ butter resulted in reduction of body weight (BW) gain within 2 days, accompanying with significantly lowered adipose tissue weight [15]. Zhang et al. reported that visceral fat area, $\mathrm{BW}$, and body fat were reduced significantly by drinking a 350-mL bottle of catechin-enriched green tea beverage which contained $609.3 \mathrm{mg}$ total catechins and $68.7 \mathrm{mg}$ caffeine after lunch time daily for 12 weeks in Chinese adults [16].

The preventive mechanism of tea on obesity was related to inhibition of pancreatic lipase (PL) and regulation of expressions of proteins or related genes. Grove et al. reported that supplementation with feed containing $0.32 \%$ green tea EGCG for 6 weeks in high fat-fed, obese mice, BW gain and fecal lipid content decreased by $44 \%(p<0.01)$ and $29.4 \%(p<0.05)$ compared to control, whereas in vitro, EGCG dose-dependently inhibited PL (IC50 = $7.5 \mathrm{mu} \mathrm{mol} / \mathrm{l})$ in a noncompetitive manner with respect to substrate concentration [17]. Insulin-like growth factor binding protein-1 (IGFBP-1) was considered to be a novel molecule target for the prevention of obesity by green tea in adipose tissue [18]. In C57BL/6 mice fed a high-fat (HF) diet and 3T3-L1 adipocytes, treatment with green tea and its major polyphenol EGCG induced the expression of IGFBP-1 and the IGFBP-1 expression level was negatively correlated with adipose tissue weight. Lu et al. revealed the beneficial effects of GTP on BW via regulating obesity-related genes, anti-inflammation, anti-oxidant capacity, and estrogen-related actions in HF-induced obese SD rats. The expression levels of orexigenic genes (Agrp, Ghrl, and $\mathrm{Nr3c1}$ ), anorectic genes (Apoa4, Cntf, Ghr, IL-1 beta, Ins1, Lepr, and Sort) and genes relating to energy expenditure (Adcyap1r1 and Adrb1) were significantly changed by feeding with HF-diet, but supplementation of GTP in the drinking water restored the expression levels of these genes and reduced BW as compared to the HF-diet group [19].

The major inflammatory cytokines interleukins (IL) such as IL6, play a crucial role in infection, inflammation and stress responses. Matrix metalloproteinases (MMPs) consist of a multigene family of zinc-dependent extracellular matrix (ECM) remodeling endopeptidases implicated in pathological processes. The mRNA expression of both IL-6 and MMP-9 increased significantly in a time-dependent manner in THP-1 macrophages cultured in normocholesterolaemic hypertensive sera $(\mathrm{p}<0.05)$, but the expression increase was abolished by GTP treatment $(p<0.05)[20]$.

It is known that plasminogen activator inhibitor-1 
(PAI-1) plays a pivotal role in CVD including arteriosclerosis and hypertension. The high PAI-1 plasma levels were associated with coronary heart disease. Cardiovascular protection effects of GTP are considered to be related to the GTP inhibition of PAI-1 expression and PAI-1 release from endothelial cells through the PI3K/ Akt pathway. Liu et al. showed that the expression and secretion of PAI-1 in endothelial cells and bovine aortic endothelial cells were significantly reduced by GTP treatment in a time dependent and dose-dependent manner. The GTP-induced inhibitory effect was associated with an increased activation of the protein kinase Akt [21].

Caveolin-1 (Cav-1), a negative regulator of endothelial nitric oxide synthase (eNOS), influences various aspects of the cardiovascular functions. GTP $(0.04-4.00 \mu \mathrm{g} / \mathrm{mL})$ down-regulated Cav-1 protein expressions and mRNA levels dose-dependently in bovine aortic endothelial cells (BAECs) via activating ERK1/2 and inhibiting p38MAPK signaling [22].

Hypercholesterolemia is the presence of higher concentrations of low density lipoprotein (LDL) and lower concentrations of functional high density lipoprotein (HDL) in the blood, which are strongly associated with CVD because these promote atheroma development in arteries (atherosclerosis). A study on obese women showed that oral administration of a capsule containing $400 \mathrm{mg}$ green tea extract three times per day for 12 weeks had significant reduction in LDL-cholesterol and triglyceride, but marked increase in the levels of HDL-cholesterol, adiponectin and ghrelin, though the decrease in BW was not statistically significant [23].

Diabetes and obesity are common metabolic disorders which are associated with the development of CVD. Moderate tea consumption ( 1 - 2 cups/day) was associated with $88 \%$ (95\% CI 76\% - 98\%) lower odds of having diabetes among non-obese participants, irrespective of age, sex, smoking, physical activity status, dietary habits and other clinical characteristics [24].

\section{INHIBITING ANGIOTENSIN CONVERTING ENZYME}

Angiotensin is a peptide hormone that causes vasoconstriction and a subsequent increase in blood pressure, and it is a part of the renin-angiotensin system, which is a major target for drugs that lower blood pressure. It is considered that the effect of green tea on hypertension might be related to its inhibition of angiotensin converting enzyme (ACE). Liang et al. showed that intragastric administration at dosages 0.2 and $1.0 \mathrm{~g} / \mathrm{kg} \cdot \mathrm{d}$ of green tea significantly decreased blood pressure and plasma angiotensin II level, accompanying the improvement of ultra structures of left ventricular mitochondrion and myofibrillae in SHR [25]. The inhibitory effect of tea on ACE depended on chemical composition of the tea. It was reported that green tea prepared using leaf of tea cultivar "Benifuuki" had a stronger suppressive effect on the ACE activity and blood pressure because it contained a higher level of (-)-epigallocatechin-3-O-(3-O-methyl) gallate, an O-methylated derivative of EGCG than the other cultivars [26]. The GABA (gamma-aminobutyric acid)-rich tea was more effective in preventing the development of hypertension in Dahl salt-sensitive rats fed with a high salt diet [27].

\section{IMPROVING ENDOTHELIAL DYSFUNCTION}

Endothelial dysfunction is a systemic disorder and a key variable in the pathogenesis of atherosclerosis and it is regarded as an integrated index of all atherogenic and atheroprotective factors present in an individual.

Black tea consumption significantly increased the posthyperemia brachial artery diameter (BAD), flow-mediated vasodilation (FMV), and FMV\% $(\mathrm{p}<0.05)$, resulting in improvement of endothelial function and endothelium-dependent arterial vasodilation in renal transplant recipients [28]. Ihm et al. found that intake of catechin normalized blood pressure and prevented endothelial dysfunction and IR in the prediabetic stage [7].

A key modulator of endothelial cell activity is nitric oxide (NO), which under physiological conditions is mainly produced by the endothelial nitric oxide synthase (eNOS) isoform. NO regulates vascular tone, proliferation of vascular smooth muscle cells and hemostasis. Disruptions in the physiological production of NO triggers endothelial cell dysfunction, resulting in an increased risk of CVD. Treatment of human coronary artery endothelial cells with green tea EC led to time- and dose-dependent effects on eNOS, peaking at $10 \mathrm{~min}$ at 1 $\mu \mathrm{mol} / \mathrm{L}$. EC treatment activated eNOS via serine 633 and serine 1177 phosphorylation, threonine 495 dephosphorlation and partially via the $\mathrm{Ca}^{2+} / \mathrm{CaMKII}$ pathway [29]. The physiological increase in NO bioavailability by green tea is considered to be promising for the prevention and therapy of CVD.

\section{PREVENTING CARDIAC HYPERTROPHY}

Cardiac hypertrophy is a thickening of the heart muscle (myocardium) which results in a decrease in size of the chamber of the heart, including the left and right ventricles. It is usually the response to stress or disease such as hypertension, heart muscle injury, heart failure or neurohormones. GTE might block the development of cardiac hypertrophy in an animal model of chronic renal failure. Male SD rats were subjected to sham or remnant kidney 
surgery and given green tea extract (GTE, $0.1 \%$ and $0.25 \%$ ) or plain drinking water for the next 4 weeks. The administration of GTE at $0.25 \%$ resulted in attenuation of left ventricular hypertrophy, hypertension, and preserved cardiac Na-K-ATPase activity in rats subjected to remnant kidney surgery $(\mathrm{p}<0.01)$. GTE prevented increases in ROS production [30].

A critical event in the development of atherosclerosis, hypertension, and angioplasty-induced restenosis is the hypertrophy of vascular smooth muscle cell (VSMC), which can be stimulated by Angiotensin II (Ang II). There was study showed that EGCG inhibited Ang IIstimulated VSMC hypertrophy through the JNK signaling pathway at both transcriptional and posttranslational levels [31].

Prevention of cardiac remodeling due to increased pressure overload is important to reduce morbidity and mortality. EGCG prevented the development of left ventricular concentric hypertrophy by pressure overload. Cardiac hypertrophy can be induced by suprarenal transverse abdominal aortic constriction (AC) in rats. Experiment showed that after 3 weeks of AC surgery, heart to body weight ratio increased in the AC group by $34 \%$ compared to the sham group, while EGCG administration suppressed the load-induced increase in heart weight by $69 \%$ [32], suggesting that increased left ventricular systolic dimensions and deteriorated systolic function were relieved by EGCG.

Green tea both prevented and reversed the cardiovascular remodeling and metabolic changes seen in high carbohydrate-fed rats. High carbohydrate diet-fed rats showed glucose intolerance, hypertension, mild left ventricular hypertrophy and etc. Administration of green tea to high carbohydrate diet-fed rats prevented and reversed glucose intolerance and the increased systolic blood pressure, left ventricular wet weight, interstitial collagen and passive diastolic stiffness [33].

\section{PROTECTING MITOCHONDRIA FROM DAMAGE}

Mitochondria are described as "cellular power plants" and mitochondrial disorders are implicated in the development of cardiac dysfunctions. Green tea gavage at dosage $0.2-2.0 \mathrm{~g} / \mathrm{kg} \cdot$ day protected mitochondria of left ventricular myocardium and aortic vascular smooth muscle cells from damage in SHR, along with decline in SBP and the levels of plasma nitric oxide, aldosterone, malondialdehyde, and left ventricular hypertrophy index (LHVI) in a dose dependant manner [34]. Transaortic abdominal aortic constriction (TAC) decreased mitochondrial DNA copy number and the activity of respiratory chain complexes I, III and IV in rats, but these decreases could be reversed by EGCG treatment [35].
Mitochondrial DNA 5178 cytosine/adenine (Mt5178C/A) polymorphism is reported to be associated with longevity and to modify the effects of alcohol consumption on the risk of hypertension in the Japanese population. Kokaze et al. reported that there was a joint effect for $\mathrm{Mt} 5178 \mathrm{C} / \mathrm{A}$ polymorphism and green tea consumption on the risk of hypertension in middle-aged Japanese men. Irrespective of antihypertensive drug treatment, the association between Mt5178C genotype and hypertension was dependent on green tea consumption [36].

\section{CONFLICTING RESULTS}

There were conflicting results about the effect of drinking of tea on CVD and BP [37]. A case-control study carried out among nulliparous pregnant women in Quebec, Canada showed that persistent tea drinking during pregnancy may be associated with an increased risk of pre-eclampsia [38]. There were studies of tea consumption involving a total of 343 subjects with a duration of 4 weeks showing that the tea intake had no significant effects on blood pressure compared with controls [39]. Some studies even showed that tea ingestion caused larger acute increases in blood pressure than caffeine alone, but any acute pressor effect of tea ingestion on blood pressure was transient [40].

There are many factors which affect the effects of tea on cardiovascular diseases and blood pressure. The effects of tea on blood pressure may be varied in different populations such as smokers, diabetics and those with various elevations in blood pressure. This may explain why conflicting results were given by various researchers who did the studies under different backgrounds. Many epidemiological surveys were carried out in the population of non-smokers who had high-normal blood pressures or mild systolic hypertension [40]. However, animal tests were carried out on the spontaneously hypertensive rats (SHR) $[8,11,13,21]$. The dosages used were quite different between the tests, for example, green tea $7.6 \mathrm{~g}$ per person per day in human test [40], but $0.2-1.0$ $\mathrm{g} / \mathrm{kg} \cdot$ day in SHR test [25]. Many experiments reported were carried out in a short-term period, and the shortterm study may not reveal the longer-term interventions and long-term consumption of tea. In addition, poor oral bioavailability of tea catechins might be an important factor leading to low pharmaceutic effects of tea ingestion [41-43]. Differences in oral bioavailability of tea catechins between individuals with various physiological statuses might lead to the different responses to tea and tea extracts.

\section{CONCLUSIONS}

Tea is rich in antioxidant catechins which have effects suppressing ROS and NADPH, but stimulating eNOS, 


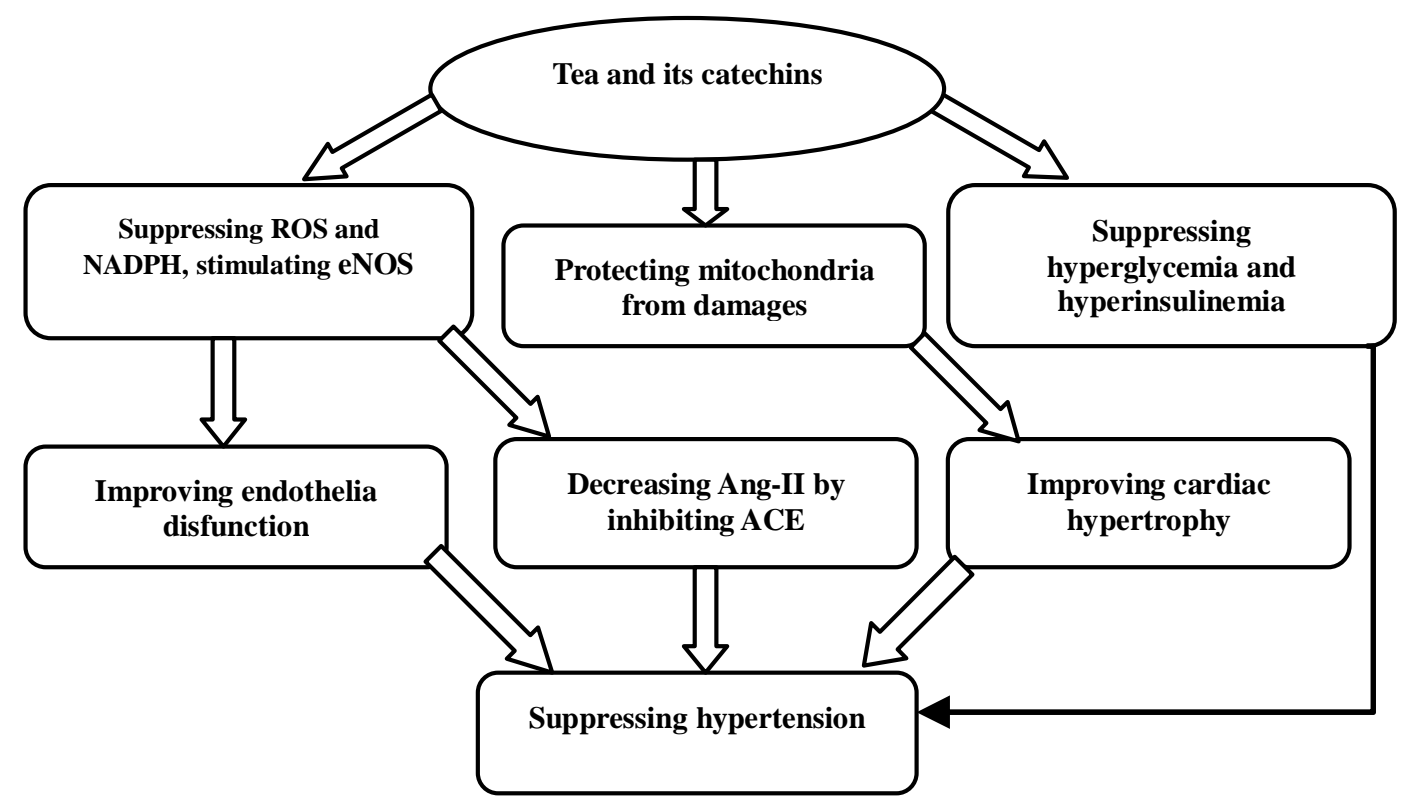

ROS: reactive oxygen species; NADPH: nicotinamide adenine dinucleotide phosphate; Ang-II: angiotensin II; ACE: angiotensin converting enzyme.

Figure 1. Actions of tea and catechins on the development of CVD and hypertension.

resulting in improvement of endothelia disfunction and decrease in Ang-II. Tea catechins improved cardiac hypertrophy through its protecting organelle, such as mitochondria from damages. Tea and catechins also suppressed hyperglycemia and hyperinsulinemia. All of these functions would contribute to the suppression of hypertension (Figure 1).

The conflicting results reported about the effects of tea and catechins on CVD might be attributed to the differences in research conditions such as dosages and physiological status of tested subjects, and also different bioavailability between the tested individuals. Further studies on the absorption mechanism of tea catechins by human body and techniques to improve their oral bioavailability will be prospective aspects in this filed.

\section{ACKNOWLEDGEMENTS}

This work was financially supported by National Key Technology R\&D Program in the 12th Five Year Plan of China (Project No. 2012BAD36B06-2).

\section{REFERENCES}

[1] Graham, H.N. (1992) Green tea composition, consumption, and polyphenol chemistry. Preventive Medicine, 21, 334-350. doi:10.1016/0091-7435(92)90041-F

[2] Yang, Y.C., Lu, F.H., Wu, J.S., Wu, C.H. and Chang, C.J. (2004) The protective effect of habitual tea consumption on hypertension. Archives of Internal Medicine, 164, 15341540. doi:10.1001/archinte.164.14.1534

[3] Mineharu, Y., Koizumi, A., Wada, Y., Iso, H., Watanabe,
Y., Date, C., Yamamoto, A., Kikuchi, S., Inaba, Y., Toyoshima, H., Kondom, T. and Tamakoshim, A. (2011) Coffee, green tea, black tea and oolong tea consumption and risk of mortality from cardiovascular disease in Japanese men and women. Journal of Epidemiology and Community Health, 65, 230-240. doi:10.1136/jech.2009.097311

[4] Wang, Q.M., Gong, Q.Y., Yan, J.J., Zhu, J., Tang, J.J., Wang, M.W., Yang, Z.J. and Wang, L.S. (2010) Association between green tea intake and coronary artery disease in a Chinese population. Circulation Journal, 74, 294300. doi:10.1253/circj.CJ-09-0543

[5] Yang, Y.J., Kim, Y.J., Yang, Y.K., Kim, J.Y. and Kwon, O. (2012) Dietary flavan-3-ols intake and metabolic syndrome risk in Korean adults. Nutrition Research and Practice, 6, 68-77. doi:10.4162/nrp.2012.6.1.68

[6] Lin, S.D., Liang, C.H., Liu, E.H. and Mau, J.L. (2010) Antioxidant properties of water extracts from parching green tea. Journal of Food Biochemistry, 34, 477-500.

[7] Ihm, S.H., Jang, S.W., Kim, O.R., Chang, K., Oak, M.H., Lee, J.O., Lim, D.Y. and Kim, J.H. (2012) Decaffeinated green tea extract improves hypertension and insulin resistance in a rat model of metabolic syndrome. Atherosclerosis, 224, 377-383.

doi:10.1016/j.atherosclerosis.2012.07.006

[8] Negishi, H., Xu, J.W., Ikeda, K., Njelekela, M., Nara, Y. and Yamori, Y. (2004) Black and green tea polyphenols attenuate blood pressure increases in stroke-prone spontaneously hypertensive rats. Journal of Nutrition, 134, 38-42.

[9] Ying, C.J., Xu, J.W., Ikeda, K., Takahashi, K., Nara, Y. and Yamori, Y. (2003) Tea polyphenols regulate nicotinamide adenine dinucleotide phosphate oxidase subunit expression and ameliorate angiotensin II-induced hyperpermeability in endothelial cells. Hypertension Research, 


\section{6, 823-828. doi:10.1291/hypres.26.823}

[10] Jówkoa, E., Sacharukb, J., Balasińskac, B., Ostaszewskic, P., Charmasa, M. and Charmasa, R. (2011) Green tea extract supplementation gives protection against exerciseinduced oxidative damage in healthy men. Nutrition Research, 31, 813-821.

[11] Wang, M.H., Chang, W.J., Soung, H.S. and Chang, K.C. (2012) (-)-Epigallocatechin-3-gallate decreases the impairment in learning and memory in spontaneous hypertension rats. Behavioural Pharmacology, 23, 771-780. doi:10.1097/FBP.0b013e32835a3bc8

[12] Wu, L.Y., Juan, C.C., Hwang, L.S., Hsu, Y.P., Ho, P.H. and Ho, L.T. (2004) Green tea supplementation ameliorates insulin resistance and increases glucose transporter IV content in a fructose-fed rat model. European Journal of Nutrition, 43, 116-124. doi:10.1007/s00394-004-0450-x

[13] Potenza, M.A., Marasciulo, F.L., Tarquinio, M., Tiravanti, E., Colantuono, G., Federici, A., Kim, J.A., Quon, M.J. and Montagnani, M. (2007) EGCG, a green tea polyphenol, improves endothelial function and insulin sensitivity, reduces blood pressure, and protects against myocardial I/R injury in SHR. American Journal of Physiology-Endocrinology and Metabolism, 292, 1378-1387. doi:10.1152/ajpendo.00698.2006

[14] Bogdanski, P., Suliburska, J., Szulinska, M., Stepien, M., Pupek-Musialik, D. and Jablecka, A. (2012) Green tea extract reduces blood pressure, inflammatory biomarkers, and oxidative stress and improves parameters associated with insulin resistance in obese, hypertensive patients. Nutrition Research, 32, 421-427. doi:10.1016/j.nutres.2012.05.007

[15] Hasegawa, N., Yamda, N. and Mori, M. (2003) Powdered green tea has antilipogenic effect on zucker rats fed a high-fat diet. Phytotherapy Research, 17, 477-480. doi:10.1002/ptr.1177

[16] Zhang, Y., Yu, Y., Li, X., Meguro, S., Hayashi, S., Katashima, M., Yasumasu, T. and Li, K. (2012) Effects of catechin-enriched green tea beverage on visceral fat loss in adults with a high proportion of visceral fat: A double-blind, placebo-controlled, randomized trial. Journal of Functional Foods, 4, 315-322. doi:10.1016/j.jff.2011.12.010

[17] Grove, K.A., Sae-tan, S., Kennett, M.J. and Lambert, J.D. (2012) (-)-Epigallocatechin-3-gallate inhibits pancreatic lipase and reduces body weight gain in high fat-fed obese mice. Obesity, 20, 2311-2313. doi:10.1038/oby.2011.139

[18] Ueda, M. and Ashida, H. (2012) Green tea prevents obesity by increasing expression of insulin-like growth factor binding protein-1 in adipose tissue of high-fat diet-fed mice. Journal of Agricultural and Food Chemistry, 60, 8917-8923. doi:10.1021/jf2053788

[19] Lu, C.W., Zhu, W.B., Shen, C.L. and Gao, W.M. (2012) Green tea polyphenols reduce body weight in rats by modulating obesity-related genes. Plos One, 7, e38332. doi:10.1371/journal.pone.0038332

[20] Mahajan, N., Dhawan, V., Sharma, G., Jain, S. and Kaul, D. (2008) Induction of inflammatory gene expression by THP-1 macrophages cultured in normocholesterolaemic hypertensive sera and modulatory effects of green tea polyphenols. Journal of Human Hypertension, 22, 141143. doi:10.1038/sj.jhh.1002277

[21] Liu. J., Ying, C., Meng, Y., Yi, W., Fan, Z., Zuo, X., Tian, C. and Sun, X. (2009) Green tea polyphenols inhibit plasminogen activator inhibitor-1 expression and secretion in endothelial cells. Blood Coagulation and Fibrinolysis, 20, 552-557. doi:10.1097/MBC.0b013e32832e 05f0

[22] Li, Y.R., Ying, C.J., Zuo, X.Z., Yi, H.W., Yi, W.J., Meng, Y., Ikeda, K., Ye, X.L., Yamori, Y. and Sun, X.F. (2009) Green tea polyphenols down-regulate caveolin-1 expression via ERK1/2 and p38MAPK in endothelial cells. Journal of Nutritional Biochemistry, 20, 1021-1027. doi:10.1016/j.jnutbio.2008.12.001

[23] Hsu, C.H., Tsai, T.H., Kao, Y.H., Hwang, K.C., Tseng, T.Y. and Chou, P. (2008) Effect of green tea extract on obese women: A randomized, double-blind, placebocontrolled clinical trial. Clinical Nutrition, 27, 363-370. doi:10.1016/j.clnu.2008.03.007

[24] Polychronopoulos, E., Zeimbekis, A., Kastorini, C.M., Papairakleous, N., Vlachou, I., Bountziouka, V. and Panagiotakos, D.B. (2008) Effects of black and green tea consumption on blood glucose levels in non-obese elderly men and women from Mediterranean Islands (MEDIS epidemiological study). European Journal of Nutrition, 47, 10-16. doi:10.1007/s00394-007-0690-7

[25] Liang, Y.R., Xu, J.Y., Luo, X.Y., Zheng, X.Q., Sun, Q.L., Ma, S.C. and Lu, J.L. (2010) Effect of green tea on angiotensin II level and myocardial microstructure in spontaneous hypertensive rats. Journal of Medicinal Plants Research, 4, 1843-1846.

[26] Kurita, I., Maeda-Yamamoto, M., Tachibana, H. and Kamei, M. (2010) Antihypertensive effect of Benifuuki tea containing O-methylated EGCG. Journal of Agricultural and Food Chemistry, 58, 1903-1908. doi:10.1021/jf904335g

[27] Abe, Y., Umemura, S., Sugimoto, K., Hirawa, N., Kato, Y., Yokoyama, N., Yokoyama, T., Iwai, J. and Ishii, M. (1995) Effect of green tea rich in gamma-aminobutyricacid on blood-pressure of Dahl salt-sensitive rats. American Journal of Hypertension, 8, 74-79. doi:10.1016/0895-7061(94)00141-W

[28] Ardalan, M.R., Tarzamni, M.K., Shoja, M.M., Tubbs, R.S., Rahimi-Ardabili, B., Ghabili, K. and Khosroshahi H.T. (2007) Black tea improves endothelial function in renal transplant recipients. Transplantation Proceedings, 39, 1139-1142. doi:10.1016/j.transproceed.2007.04.010

[29] Ramirez-Sanchez, I., Maya, L., Ceballos, G. and Villarreal, F. (2010) (-)-Epicatechin activation of endothelial cell endothelial nitric oxide synthase, nitric oxide, and related signaling pathways. Hypertension, 55, 1398-1405. doi:10.1161/HYPERTENSIONAHA.109.147892

[30] Priyadarshi, S., Valentine, B., Han, C., Fedorova, O.V., Bagrov, A.Y., Liu, J., Periyasamy, S.M., Kennedy, D., Malhotra, D., Xie, Z. and Shapiro, J.I. (2003) Effect of green tea extract on cardiac hypertrophy following $5 / 6$ nephrectomy in the rat. Kidney International, 63, 17851790. doi:10.1046/j.1523-1755.2003.00914.x 
[31] Zheng, Y., Song, H.J., Kim, C.H., Kim, H.S., Kim, E.G., Sachinidis, A. and Ahn, H. (2004) Inhibitory effect of epigallocatechin 3-O-gallate on vascular smooth muscle cell hypertrophy induced by angiotensin II. Journal of Cardiovascular Pharmacology, 43, 200-208. doi:10.1097/00005344-200402000-00006

[32] Hao, J., Kim, C.H., Ha, T.S. and Ahn, H.Y. (2007) Epigallocatechin-3-gallate prevents cardiac hypertrophy induced by pressure overload in rats. Journal of Veterinary Science, 8, 121-129. doi:10.4142/jvs.2007.8.2.121

[33] Rickman, C., Iyer, A., Chan, V. and Brown, L. (2010) Green tea attenuates cardiovascular remodeling and metabolic symptoms in high carbohydrate-fed rats. Current Pharmaceutical Biotechnology, 11, 881-886. doi:10.2174/138920110793261980

[34] Liang, Y.R., Ma, S.C., Luo, X.Y., Xu, J.Y., Wu, M.Y., Luo, Y.W., Zheng, X.Q. and Lu, J.L. (2011) Effects of green tea on blood pressure and hypertension-induced cardiovascular damage in spontaneously hypertensive rat. Food Science and Biotechnology, 20, 93-98. doi:10.1007/s10068-011-0013-x

[35] Chen, D.D., Dong, Y.G., Liu, D. and He, J.G. (2009) Epigallocatechin-3-gallate attenuates cardiac hypertrophy in hypertensive rats in part by modulation of mitogen-activated protein kinase signals. Clinical and Experimental Pharmacology and Physiology, 36, 925-932. doi:10.1111/j.1440-1681.2009.05173.x

[36] Kokaze, A., Ishikawa, M., Matsunaga, N., Karita, K., Yoshida, M., Ohtsu, T., Ochiai, H., Shirasawa, T., Saga, N., Hoshino, H. and Takashima, Y. (2012) Combined effect of longevity-associated mitochondrial dna $5178 \mathrm{c} / \mathrm{a}$ polymorphism and green tea consumption on risk of hypertension in middle-aged Japanese men. Human Biology, 84, 307-318. doi:10.3378/027.084.0309
[37] Watson, R.E., Karnchanasorn, R. and Gossain, V.V. (2009) Hypertension in Asian Pacific Island Americans. The Journal of Clinical Hypertension, 11, 148-152. doi:10.1111/j.1751-7176.2009.00088.x

[38] Wei, S.Q., Xu, H., Xiong, X., Luo, Z.C., Audibert, F. and Fraser, W.D. (2009) Tea consumption during pregnancy and the risk of pre-eclampsia. International Journal of Gynecology and Obstetrics, 105, 123-126. doi:10.1016/j.ijgo.2008.12.003

[39] Taubert, D., Roesen, R. and Schomig, E. (2007) Effect of cocoa and tea intake on blood pressure: A meta-analysis. Archives of Internal Medicine, 167, 626-634. doi:10.1001/archinte.167.7.626

[40] Hodgson, J.M., Puddey, I.B., Burke, V., Beilin, L.J. and Jordan, N. (1999) Effects on blood pressure of drinking green and black tea. Journal of Hypertension, 17, 457463. doi:10.1097/00004872-199917040-00002

[41] Zhang, L., Zheng, Y., Chow, M.S.S. and Zuo, Z. (2004) Investigation of intestinal absorption and disposition of green tea catechins by Caco-2 monolayer model. International Journal of Pharmaceutics, 287, 1-12. doi:10.1016/j.ijpharm.2004.08.020

[42] Tagashira, T., Choshi, T., Hibino, S., Kamishikiryou, J. and Sugihara, N. (2012) Influence of gallate and pyrogallol moieties on the intestinal absorption of (-)-epicatechin and (-)-epicatechin gallate. Journal of Food Science, $77,208-215$. doi:10.1111/j.1750-3841.2012.02902.x

[43] Hu, B., Ting, Y.W., Zeng, X.X. and Huang, Q.R. (2012) Cellular uptake and cytotoxicity of chiosan-caseino-phosphorpeptides nanocomplexes loaded with epigallocatechin gallate. Carbohydrate Polymers, 89, 362-370. doi:10.1016/j.carbpol.2012.03.015 